\title{
Effort or Escape: Self-concept Structure Determines Self-regulatory Behavior
}

\author{
CHRISTINA M. BROWN \\ ALLEN R. MCCONNELL \\ Miami University, Oxford, Ohio, USA
}

\begin{abstract}
The current research examined the interaction between self-complexity, affect, and perceived goal attainability on self-regulation. Participants received failure feedback about their performance and were given the opportunity to reduce this discrepancy through practice. Greater self-complexity was associated with greater practice, regardless of affect and perceived effectiveness of practice. Lower self-complexity was only associated with greater practice when participants experienced negative affect and believed practice was effective at improving performance. These results suggest that those greater in self-complexity self-regulate when external cues signal the presence of a self-discrepancy, whereas those lower in self-complexity selfregulate when negative affect signals the presence of a discrepancy. However, when the discrepancy cannot be reduced directly, lower self-complexity individuals escape the situation to avoid negative affect.
\end{abstract}

Keywords: Affect; Self-concept; Self-complexity; Self-regulation.

The ability to regulate one's behavior in pursuit of goals is a hallmark of successful human functioning. For example, individuals who cannot regulate their actions are censured by others (e.g., Vohs \& Ciarocco, 2004) and those who perceive their goals as unattainable experience negative emotions (e.g., Higgins, 1987). Despite the clear benefits of successful self-regulation, empirical studies often find that the majority of individuals report some discrepancy between their current state and how they feel they "ought" to be (Strauman \& Higgins, 1987), indicating that self-regulation is not always successful at bringing about desired end states. Thus, it is important to understand factors contributing to successful and unsuccessful attempts at self-regulation.

Recently, researchers have identified a variety of factors, such as regulating negative emotions at the expense of other goal pursuits (e.g., Tice, Bratslavasky, \& Baumeister, 2001) and the availability of glucose (e.g., Gailliot \& Baumeister, 2007), that influence effective self-regulation. Perhaps the most extensively studied component of selfregulation is current affect. Most classic theories of self-regulation (e.g., Carver \& Scheier, 1982; Higgins, 1987; Markus \& Nurius, 1986) propose that a discrepancy between one's current state and a goal state produces negative affect. This negative affect is generally believed to signal the presence of a discrepancy, leading the individual

Received 25 March 2008; accepted 18 July 2008; first published online 20 October 2008.

This work supported by NSF grant BCS 0601148 and by the Lewis Endowed Professorship.

Correspondence should be addressed to: Christina M. Brown, Department of Psychology, Saint Louis University, Shannon Hall 111, 3511 Laclede Ave., Saint Louis, MO 63101, USA.

E-mail: cbrown81@slu.edu 
to initiate and direct goal-relevant behaviors. Once the goal is reached (or the individual makes sufficient progress toward it; Carver, 2004), positive affect replaces the negative affect, and self-regulatory efforts are reduced or terminated. However, the specific mechanism by which negative affect governs self-regulation is less clear, with some theorists proposing that it merely informs the individual of a discrepancy (e.g., Carver, 2004), while others contend that the desire to eliminate unpleasant affect directly motivates regulatory behavior (e.g., Duval \& Wicklund, 1972).

Importantly, negative affect does not exist in a vacuum and is often intricately connected to other aspects of the self. For example, the type of negative affect (e.g., dejection vs. agitation) experienced can depend on the type of self-discrepancy (e.g., Higgins, 1987; cf. Phillips \& Silvia, 2005) and people's lay theories of personality influence how self-discrepancies impact self-esteem (e.g., Renaud \& McConnell, 2007). More germane to the current work, research has established that the structure of the individual's self-concept can influence the experience of affect. We now turn our attention to one such feature, self-complexity.

\section{Self-complexity and Affect}

According to self-complexity theory (see Linville, 1985; McConnell \& Strain, 2007), the self is comprised of multiple self-aspects, each representing a meaningful domain of one's life (e.g., doctor, spouse, athlete). A person who possesses many different self-aspects that are comprised of unique and differentiated attributes (e.g., cooperative as a spouse but competitive as an athlete) is said to be greater in selfcomplexity than an individual with fewer self-aspects that share more attributes in common (e.g., competitive as a doctor, spouse, and athlete).

An important implication of attribute overlap among self-aspects is that when success or failure is experienced with respect to a self-aspect, other self-aspects sharing common attributes are also affected (Linville, 1985). In contrast, when selfrelevant feedback targets a self-aspect that shares little or no overlap of attributes with other self-aspects, it has less impact on affect because the feedback stays confined to the targeted self-aspect. That is, spreading activation among self-aspects is less likely to occur because there are fewer associative links among self-aspects for the feedback to be propagated throughout one's self-concept. This effect, that people lower in self-complexity experience stronger reactions than those greater in selfcomplexity, is known as affective spillover. Affective spillover has been found in response to positive and negative feedback (Linville, 1985; Niedenthal, Setterlund, \& Wherry, 1992), and it contributes to stronger fluctuations in mood (Linville, 1985) and greater difficulty in suppressing self-relevant thoughts (Renaud \& McConnell, 2002) among those lower in self-complexity. In the current work, we considered whether the differential affective experiences of individuals who vary in selfcomplexity might reveal consequences for self-regulatory behavior.

\section{Self-complexity and Self-regulation}

The role of self-complexity in self-regulation was explored by Dixon and Baumeister (1991). In their study, participants who had received either success or failure feedback were told to write an essay in a room with a large mirror, which either faced them (high self-focus) or was turned away from them (low self-focus). In the high self-focus condition, those lower in self-complexity who received failure feedback finished the essay and left the room more quickly than those 
greater in self-complexity, suggesting that being lower in self-complexity made it more difficult to cope with failure under heightened self-awareness. Interestingly, the researchers found no relation between self-complexity and reported affect in their study, suggesting that the relation between self-complexity and affect in selfregulation is more complex than previously realized.

It is important to note that the conventional view of self-regulation assumes that individuals experiencing a self-discrepancy will work to reduce the discrepancy unless they believe that it is impossible to eliminate the discrepancy or if its saliency can be reduced through other means (e.g., reducing self-awareness; Carver, Blaney, \& Scheier, 1979; Duval, Duval, \& Mullilis, 1992). Dixon and Baumeister's (1991) interpretation of the behavior of those lower in self-complexity was that they chose to escape self-awareness more quickly because a greater proportion of their selfconcept was affected by the failure. However, if an opportunity to reduce the discrepancy was available, might those lower in self-complexity have exerted effort rather than escaping? The current work explored this prediction.

One remaining puzzle of Dixon and Baumeister's study is that although individuals lower in self-complexity (relative to those greater in self-complexity) escaped self-awareness more quickly, they did not report experiencing greater negative affect. These results raise the possibility that self-complexity may influence how negative affect is interpreted and used, even in circumstances where it does not directly predict the intensity of affect. Further, the relations between self-complexity, affect experienced, and subsequent self-regulatory behavior may hinge on how the affect is interpreted by the perceiver.

For instance, consider what might happen in a situation where individuals varying in self-complexity feel equally negative affect. Would these individuals display similar forms of goal-directed or withdrawal behavior? Or, on other hand, might these individuals differentially interpret and react to that negative affect? For example, affect may be more salient in the lives of those with lower self-complexity because of their more frequent and intense mood fluctuations following feedback (Linville, 1985), and consequently, their behavior may be more strongly guided by affect because of its information value. Consistent with mood as information (e.g., Schwarz \& Clore, 2003) and mood maintenance (e.g., Isen, 1987) approaches, these individuals should only self-regulate when experiencing negative affect (i.e., they interpret the negative affect as signaling a problem or discrepancy) and should not self-regulate when feeling positive (i.e., positive affect signals self-regulation is unnecessary and they should be motivated to maintain this positive affect by avoiding effort). In contrast, because the affective experiences of those greater in self-complexity are less variable (e.g., feedback does not have a strong effect on their feelings, and their moods change little over the course of a day), current affect may play less of a role in guiding behavior.

In support of this reasoning that affect is attended to and interpreted differently as a function of an individual's level of self-complexity, we collected preliminary data in which participants $(N=35)$ completed a measure of self-complexity and reported the extent to which they use their feelings to guide behavior. The measure of selfcomplexity was a paper-and-pencil measure based on Linville (1985). Specifically, participants were given a number of identical sheets, each of which presented a list of 40 attributes. Each sheet represented a "group" (self-aspect), and participants were instructed to create groups that represented meaningful aspects of their lives. For each group, they were told to circle those attributes that described them in that group and to provide a label for the group. These instructions were nearly identical to those used in the primary study (see Measures), with the only difference being that 
participants circled attributes on the sheets rather than "clicking and dragging" using a computer program. Self-complexity was measured using Scott's $H$ statistic, with larger scores indicating greater self-complexity (see Measures). ${ }^{1}$

To explore if self-complexity was related to how much attention people pay to affect when initiating behavior, participants completed two items from Pacini and Epstein's (1999) Rational-Experiential Inventory (REI) that directly assess the extent to which people attend to their current affect when deciding how to behave: "I generally don't depend on my feelings to help me make decisions," and "I think it is foolish to make important decisions based on feelings." The mean of participants' responses to these two items, recorded on a scale ranging from 1 (definitely not true of myself) to 5 (definitely true of myself), was significantly correlated with their selfcomplexity, $r=.39, p<.02$. That is, as self-complexity decreased, relying on one's affect to guide behavior increased. In the current work, we examined participants' experienced affect and behavior to further test the proposal that the self-regulatory actions of those lower in self-complexity are guided more by their affect whereas the self-regulatory behavior of those greater in self-complexity is guided more by other (i.e., external) cues.

\section{The Present Research}

The participants in Dixon and Baumeister's (1991) study did not have the opportunity to work toward their goal following failure, which meant individuals with lower self-complexity, who have greater difficulty mentally escaping thoughts about failure (Renaud \& McConnell, 2002), could only reduce the saliency of the self-discrepancy by escaping self-awareness. However, if an alternative course of action was available that permitted improving their current state in the wake of negative feedback, it is possible that people with lower self-complexity could have responded by exerting effort through practice rather than escaping self-awareness. To evaluate this possibility, we manipulated perceived goal attainability and made the following predictions:

1. When the goal is perceived as unattainable, practice should decrease (that is, the situation will be escaped more quickly) for those lower in self-complexity.

2. When the goal is perceived as attainable, self-complexity should be unrelated to practice because individuals lower in self-complexity can reduce the selfdiscrepancy directly (i.e., through practice) instead of resorting to escape.

In addition to failure feedback being more difficult to escape as self-complexity and goal attainability decrease, we also predicted that the self-regulatory significance of affect would differ depending on the individual's level of self-complexity. As described previously, because those with greater self-complexity experience relatively restricted affective variability (Linville, 1985) and report relying less on their affect to guide behavior (preliminary data), they should be less likely to perceive affect as an informative signal for self-regulation. In contrast, affect is more salient for those lower in self-complexity, who in turn report relying on their feelings to guide their behavior. As a result, differences in the experience of affect should lead the self-regulatory behavior (i.e., practice effort) of individuals with lower self-complexity to be guided by their internal affective state, whereas the self-regulatory behavior of individuals with greater self-complexity should be more likely to be guided by the external situation (e.g., feedback indicating a self-discrepancy). In other words, we propose that people 
with lower self-complexity should self-regulate only when negative affect signals the presence of a self-discrepancy, whereas the self-regulation of those greater in selfcomplexity should not depend on their current affect. Specifically, we predicted:

3. Because those lower in self-complexity should use their own affect as a cue for self-regulation, when affect is experienced as negative, practice effort (i.e., selfregulation) should increase as self-complexity decreases.

That is, individuals with lower self-complexity should self-regulate (i.e., reduce a discrepancy through effortful practice) only when they are experiencing negative affect, whereas individuals with greater self-complexity should self-regulate regardless of their affect, provided that there is an external cue (e.g., feedback) indicating the presence of a self-discrepancy.

To test these predictions, we first created a self-discrepancy among participants by providing failure feedback about their performance on a test that purportedly assessed an important ability. Self-regulatory effort was assessed by the degree to which participants practiced, with greater practice reflecting relatively greater goaldirected effort and less practice indicating greater escape from the situation.

\section{Method}

Participants

Participants were 76 students (32 women) at Miami University who participated in return for partial course credit. Nine participants were suspicious of the feedback and the second measure of affect, thus these participants were removed from all analyses, resulting in a total of 67 participants in the analyses (29 women).

\section{Measures}

Self-complexity. Self-complexity was measured using a computerized version of Linville's (1985) self-complexity trait-sorting task (McConnell et al., 2005). Participants were provided with a list of 20 positive and 20 negative attributes that are commonly used by college students to describe themselves (Showers, 1992), and they were asked to sort the attributes into groups (i.e., self-aspects) that represent meaningful aspects of their lives. Participants were told they did not have to use all of the attributes, that attributes could be used in more than one group, and that they should stop if they felt they were straining to generate more groups.

Self-complexity was calculated using the $H$ statistic (Scott, 1969), which captures both the number of self-aspects (i.e., groups) and the redundancy in attributes among self-aspects:

$$
H=\log _{2} n-\left(\Sigma_{i} n_{i} \log _{2} n_{i}\right) / n
$$

where $n$ is the total number of attributes available to the participant (40) and $n_{\mathrm{i}}$ is the number of attributes present within each particular group combination (i) across the participant's reported self-aspects.

Affect. The Positive and Negative Affect Schedule (PANAS; Watson, Clark, \& Tellegen, 1988) was administered via computer to measure participants' current affect. 
This measure contains 20 emotion adjectives, 10 positive (e.g., interested, proud) and 10 negative (e.g., upset, jittery). Using a scale ranging from 1 (not at all or very slightly) to 7 (extremely), participants indicated the extent to which they were currently experiencing each emotion. Responses to the negative adjectives were reverse-coded and averaged with responses to the positive adjectives, forming an index of current affect (e.g., Renaud \& McConnell, 2002). The PANAS was administered twice to obtain participants' affect before and after experimental feedback. The computer recorded participants' responses and response latencies for each item, and responses faster than $300 \mathrm{~ms}$ (indicating an accidental or random response) were removed.

\section{Procedure}

Participants were tested individually and told that they would be describing themselves on a computer task and completing tests of verbal ability. First, they were seated at a computer and completed the baseline (i.e., pre-feedback) measure of affect. Next, participants completed the self-complexity measure.

Following these computer tasks, the experimenter told participants that the second part of the study was exploring verbal ability. Participants were instructed to turn their chair around so they were facing the experimenter for the first test. The experimenter explained that they would be taking the Thorndike Anagrams Test (TAT; see Dixon \& Baumeister, 1991) to determine their level of verbal ability, which has been found to predict success in careers and personal relationships (thus making performance on it important to participants). The TAT consisted of the experimenter holding up an index card displaying an anagram, and participants were instructed to provide an answer out loud when they solved it. If they did not solve it within $10 \mathrm{~s}$, the experimenter continued to the next card. Participants saw 10 cards total, seven of which displayed insolvable anagrams. The insolvable anagrams were generated by taking solvable anagrams and adding an extra letter such that no actual words could be constructed from the anagram. All anagrams contained 5 or 6 letters.

Following the TAT, the experimenter told participants:

Hmmm. You didn't do as well as we usually expect students to do, so I guess that was pretty tough for you. Most students who take this test solve about 6 or 7 of the anagrams, and we expect the majority of people to get a minimum of 5 problems right, which is a $50 \%$. I'm going to go to the other room to enter your score into the computer. Just wait here; I'll be right back.

Approximately $45 \mathrm{~s}$ after the experimenter left the room, she returned with an embarrassed look and said:

Um, I just checked the computer, and I must've screwed something up because it looks like the very first computer program you did was not transferred to our main computer system. I don't want the grad student in charge of this experiment to find out I messed up, so do you think you could complete the first program again?

All participants agreed to complete the first computer task (the PANAS) a second time, allowing for the comparison of participants' affect before and after the failure feedback (this procedure has been used successfully in past research; e.g., Linville, 1985; Renaud \& McConnell, 2002). After obtaining their agreement, the experimenter instructed participants to answer the questions based on their feelings at the current moment instead of trying to remember their previous responses.

When participants had finished the second affect measure, the experimenter thanked them and explained that the next task would be a paper-based task 
administered in a different location in the lab. In actuality, participants were moved to this new location because it had a mirror to increase participants' self-awareness and to strengthen the impact of the feedback and the self-discrepancy (e.g., Duval \& Wicklund, 1972). Participants were then told for the first time that they would be taking a second TAT and that they would have the opportunity to practice beforehand. The practice instructions, however, were varied such that half of the participants were led to believe practice would be effective at improving their performance while the rest were told it would be ineffective.

To manipulate perceived effectiveness of practice, the experimenter introduced the practice task in a casual and non-scripted manner (effective condition in italics, ineffective condition in brackets):

In this study, we're interested in how different types of practice influence scores on the Thorndike Anagrams Test, which is used in many academic settings. We have several different forms of practice that we're examining so we can compare everyone's final scores and see if some types of practice lead to better scores than others. So, the version of practice you're going to get is not the same as what someone else may receive. We're still in the middle of the study so we don't have the final results yet, but so far it appears that this type of practice is the best [is not as good as some of the others]. Most of the people who've had this version actually see quite an improvement in their scores on the final test. [In fact, most people who've used this version do not see any improvement in their scores on the final test.] So, it seems to be the most effective at improving scores [it appears that this type of practice is not as effective as most of the others]. But again, we're still collecting data because we want to make sure that this truly is the best type [not the most effective form] of practice.

Participants were instructed to practice as much as they wanted, but the experimenter stopped them if they were still practicing after 25 minutes (and recorded the practice time as $25 \mathrm{~min}$ ). The amount of time spent practicing and the number of problems practiced were recorded. Finally, participants were thoroughly debriefed at the conclusion of the experiment, ensuring that they understood the feedback was false and that the majority of the anagrams presented during the first task were impossible to solve.

\section{Results}

\section{Affect}

Participants' affect following the failure feedback was significantly less positive $(M=4.75, S D=0.92)$ than it was at Time $1(M=5.03, S D=0.80)$, paired samples $t(63)=4.50, p<.001$, reflecting its impact on perceptions of the self. ${ }^{2}$ Change in affect was computed by subtracting Time 2 scores from Time 1 scores, such that a larger difference score indicates an increase in negative affect. Paralleling the results of Dixon and Baumeister (1991), self-complexity was not related to change in affect following feedback.

\section{Affect, Self-complexity, Practice Effectiveness, and Effort}

Time spent practicing and number of problems practiced were significantly related, $r=.565, p<.001, \alpha=.72$, so they were standardized and summed to form an index of practice effort. Practice effort was regressed on change in affect (difference score, centered), self-complexity (centered), practice condition, and all two-way and threeway interactions. There was a main effect of self-complexity, $\beta=.38, t=3.15$, $p<.05$, and a marginal effect of condition, $\beta=.22, t=1.88, p=.065$, both of 
which were qualified by a significant three-way interaction, $\beta=-.27, t=-2.13$, $p<.05$. To explore this three-way interaction, we plotted the index of practice effort on the $y$-axis, change in affect $( \pm 1 S D$ from the $M)$ on the $x$-axis, and lines depicting the practice effective and ineffective conditions for those relatively lower in self-complexity $(M-1 S D$; top panel of Figure 1) and for those relatively greater in self-complexity $(M+1 S D$; bottom panel of Figure 1). As can be seen, greater selfcomplexity (bottom panel) was always associated with considerable practice effort, whereas the practice effort associated with lower self-complexity depended on current affect and practice effectiveness (top panel).

To further understand this interaction, a median split was performed on selfcomplexity scores to compare correlations between affect and practice effort by degree of self-complexity and condition. For low self-complexity participants in the effective condition, change in affect and practice effort were significantly correlated, $r=.53, p<.05$, revealing that those with relatively low self-complexity practiced more as their affect became especially negative following feedback (i.e., when behavior could reduce the self-discrepancy, it was guided by affect). For low selfcomplexity participants in the ineffective condition and high self-complexity participants in both conditions, there was no relation between affect and practice effort.
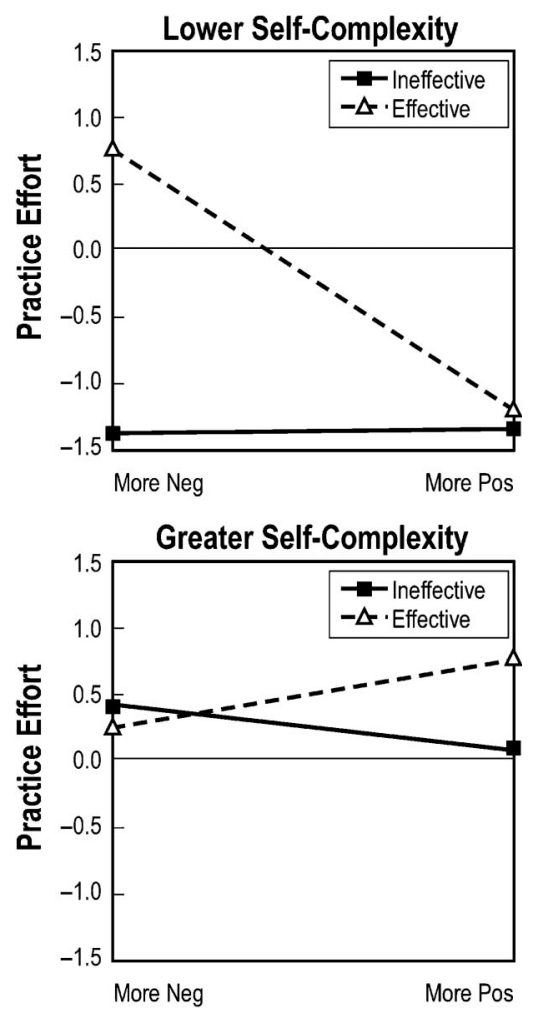

FIGURE 1 Practice effort by practice condition (effective, ineffective) and change in affect based on level of self-complexity (one standard deviation above and below the mean). 


\section{Discussion}

People with lower self-complexity regularly experience variation in affect, as demonstrated by their frequent mood fluctuations and their intense affective reactions to feedback (e.g., Linville, 1985). In addition, we found evidence that these individuals (relative to those greater in self-complexity) also rely on their affect in guiding their behavior (the preliminary data). Thus, we predicted that their behavior would be guided by their experienced affect, influencing their actions based on how their behavior might serve self-regulatory goals. Specifically, we predicted that because they attend to their affect, individuals lower in self-complexity should exert regulatory effort as long as negative affect can serve to reduce their self-discrepancy (i.e., practice is effective condition). In contrast, they should escape more quickly when there is no other method for reducing the discrepancy (i.e., practice is ineffective condition). On the other hand, the affective experience of people with greater selfcomplexity is more restricted (e.g., feedback has less effect on their feelings), so we proposed that they would be unlikely to rely on their experienced affect to guide behavior in self-regulatory situations. These predictions were confirmed, as evidenced by the three-way interaction of self-complexity, change in affect, and goal attainability.

Because they experience a limited range of affect and rely relatively little on their feelings to guide their actions, the self-regulatory behavior of people with greater self-complexity should be guided by the external situation and not by their internal affective state. The failure feedback provided by the experimenter is one such external cue, and it should be the primary force guiding the behavior of those greater in self-complexity. Indeed, in the current study, affect had little effect on the practice effort of individuals with greater self-complexity. Instead, these individuals always exerted practice effort as would be expected if they relied primarily on the external cue of the experimenter's feedback to guide their behavior (that is, the experimenter telling them they did poorly, signaling the need to change their behavior). Regardless of the perceived attainability of the goal, people with greater self-complexity practiced because the feedback from the experimenter indicated the presence of a self-discrepancy.

Consistent with multiple theories of affect such as mood maintenance (e.g., Isen, 1987) and mood as information (e.g., Schwarz \& Clore, 2003), individuals lower in self-complexity did not practice or exert effort when feeling more positive, indicating that they were attending to their current affect (i.e., they wanted to maintain the positivity or interpreted it as a signal that self-regulation was unnecessary). However, when these individuals felt more negative, they interpreted those feelings as a signal that something was wrong (i.e., they had a self-discrepancy), which would be expected if they were attending to their affect. Upon recognizing the self-discrepancy, they tried to improve their current self through effort when such an opportunity was available (i.e., practice was effective). On the other hand, when there was no way to directly reduce the discrepancy (i.e., practice was ineffective), the negative affect, which was being attended to, was unpleasant and motivated them to reduce its saliency by escaping the situation.

Because we used practice effort as an indicator of self-regulation, readers might question whether less practice truly represented escape or if it could also reflect behavioral self-handicapping (e.g., Hirt, Deppe, \& Gordon, 1991). We certainly acknowledge this alternative explanation, but we think it is unlikely for two reasons. First, Dixon and Baumeister (1991) observed that participants lower in 
self-complexity were faster to stop writing a personal essay when they were in front a mirror, which clearly represents escape from self-awareness. Our finding that speed of finishing a practice task (which occurred in front of a mirror) also increased as selfcomplexity decreased is parallel to the escape behavior observed by Dixon and Baumeister, and the parsimonious explanation would be that the same behavior was occurring (i.e., both studies involve people with lower self-complexity quickly leaving a situation of high self-awareness). Second, the least amount of practice occurred when self-complexity was low and participants believed practice was ineffective. However, withdrawing practice effort is only an effective self-handicap when individuals believe that practice can help them improve (Hirt et al., 1991), thus practice effort could not have been used as a self-handicap in the ineffective condition. Moreover, self-handicapping occurs when individuals are uncertain about their future performance (Berglas \& Jones, 1978), whereas it is highly likely that participants in the ineffective condition were certain they would do poorly on the second test.

One might also reasonably wonder about the practicality of our goal attainability manipulation. Given the commonly held belief that "practice makes perfect," it may seem challenging to manipulate the usefulness of practice. However, we believe that our specific manipulation of practice effectiveness was both believable (no participants reported disbelief or suspicion when told practice was ineffective) and provided methodological control, which can be particularly difficult to achieve for a subjective experience like "goal attainability." For example, one obvious method of manipulating goal attainability is to make participants believe that, because they performed so poorly, it is unlikely that they can improve in such a short time. However, manipulating goal attainability by emphasizing participants' inadequacies could produce a confound by reducing self-esteem and goal attainability concurrently. Another alternative would be to inform participants that the particular ability being studied tends to be stable and difficult to change (e.g., an entity theory; Dweck, Hong, \& Chiu, 1993), but this would create suspicions about the practice task by removing its rationale (i.e., participants would ask why they are being told to practice when it cannot make a difference). Considering the problems posed by alternative manipulations, we believe our approach was both effective and believable for several reasons. First, the instructions in the effective and ineffective conditions were virtually identical and all differences were balanced (providing experimental control). Second, the experimenter's rationale for the ineffective practice task was believable to participants (e.g., because skills are being tested, it is not surprising that researchers would be interested in discovering methods that improve these skills, making it inevitable that some attempts will work better than others). And, finally, and most importantly, the interaction between practice condition, self-complexity, and change in affect indicates that practice effectiveness can be and was successfully manipulated and, moreover, that it interacts with other variables to produce important outcomes.

Returning to our results, it is interesting to note that affect intensity was unrelated to level of self-complexity, paralleling the findings of Dixon and Baumeister (1991) but inconsistent with affective spillover research (e.g., Linville, 1985; Renaud \& McConnell, 2002). The participants in our and Dixon and Baumeister's studies were placed in a situation of high self-awareness (e.g., Duval \& Wicklund, 1972), which was not the case in experiments where affective spillover has been observed. It is possible that this self-focus manipulation increased the intensity of affect among people with greater self-complexity, who are usually more impervious to such effects. People with lower self-complexity are already greatly affected by feedback because of spreading activation among interrelated self-aspects, and so the self-focus 
manipulation may have had little additional impact on their already intense affect. For people with greater self-complexity, on the other hand, high self-awareness could have led them to experience affect more strongly than is usually the case (e.g., Duval \& Wicklund, 1972), which would account for the absence of a correlation between self-complexity and affect. This intense affect may have still dissipated more quickly among those with greater self-complexity, but affect was measured immediately after feedback (i.e., there was no delay) so the current work cannot test this possibility. Future research should examine the conditions (e.g., selfawareness, time course) in which self-complexity is unrelated to intensity of affect.

Based on these findings, we are compelled to question whether the selfcomplexity-affect relation is as simple as a negative correlation (those lower in self-complexity experience more intense affect). Our prediction that self-complexity influences attention to affect is based on affective spillover research (we assume that chronic variability or stability in affect produces differential responses to current affect), but there may be other aspects of the self-complexity-affect relation that are as of yet unexplored. For example, in addition to the number and uniqueness of selfaspects predicting affect, perhaps the importance of those self-aspects is influential as well. Feedback about an important and central self-aspect may produce intense affect regardless of one's self-complexity. Alternatively, the proportion of selfaspects that are relatively important may interact with self-complexity. People with greater self-complexity who see all of their self-aspects as essential to their selfdefinition may experience more intense and long-lasting affect than individuals with lower self-complexity for whom one or two self-aspects are rarely activated. At the very least, we hope that the current findings stimulate further inquiry to better understand the complex relations between self-complexity and affect.

\section{Broader Implications}

Compared to those with greater self-complexity, individuals with lower selfcomplexity are generally known to suffer more consequences following negative feedback, such as failure at thought suppression (Renaud \& McConnell, 2002) and tendencies to escape self-awareness (Dixon \& Baumeister, 1991). However, the results of the current research suggest that these individuals may not always experience such negative outcomes. As seen in this study, those lower in selfcomplexity experiencing relatively more negative affect (which signaled the presence of a self-discrepancy) tended to escape only when they believed their goal was unattainable and thus that the discrepancy could not be reduced. In contrast, these same individuals exerted goal-directed effort when they believed it was possible to reduce the self-discrepancy through effort.

These results also speak to factors influencing self-regulatory behavior. Selfregulation is an essential activity, with implications for the individual's internal affective state (Higgins, 1987) and his or her acceptance by others (Vohs \& Ciarocco, 2004). Although current affect (Carver, 2004) and goal attainability (Carver et al., 1979) are thought to affect self-regulatory behavior, the findings of the current research suggest they interact with the individual's self-concept structure to direct self-regulatory action. Specifically, those with few and overlapping self-aspects (i.e., low self-complexity) may be especially attentive to feedback and corresponding negative affect, influencing the extent to which they are driven to change a selfdiscrepancy (effort) or to merely reduce its saliency (escape). Beyond the current study, it is possible that other features of the self-concept known to influence 
approach behavior such as regulatory focus (e.g., Forster, Grant, Idson, \& Higgins, 2001), implicit theories (e.g., Dweck et al., 1993), and state self-handicapping (e.g., Strube, 1986), may also interact with affect to produce meaningful differences in selfregulation.

Thus, the relation between self-regulation and affect may be more nuanced than previously considered, as demonstrated by the moderating role of self-complexity in the current work. Given the centrality of successful self-regulation for goal attainment and behavioral pursuits, understanding how individual differences in self-concept representation influence this complex relationship has important implications for success and well-being.

\section{Notes}

1. The use of Scott's $H$ to measure self-complexity is not without controversy (e.g., Rafaeli-Mor, Gotlib, \& Revelle, 1999; Schleicher \& McConnell, 2005). In the current work, we use Scott's $H$ because it is the most widely used measure of self-complexity and, more importantly, because we sought to extend Dixon and Baumeister's (1991) findings, which used $H$ (thus allowing us to keep the measure of self-complexity consistent).

2. Degrees of freedom are different because three participants had only partial affect data. Two participants were missing Time 1 data because the experimenter overwrote the computer file containing Time 1 data when starting the Time 2 affect task. A third participant had response latencies under $300 \mathrm{~ms}$ for over half of her Time 2 responses (suggesting inattentive and random responding) and so her Time 2 responses were excluded from analyses.

\section{References}

Berglas, S., \& Jones, E. E. (1978). Drug choice as a self-handicapping strategy in response to noncontingent success. Journal of Personality and Social Psychology, 36, 405-417.

Carver, C. S. (2004). Self-regulation of action and affect. In R. F. Baumeister \& K. D. Vohs (Eds.), Handbook of self-regulation: Research, theory, and applications (pp. 13-39). New York: Guilford Press.

Carver, C. S., Blaney, P. H., \& Scheier, M. F. (1979). Reassertion and giving up: The interactive role of self-directed attention and outcome expectancy. Journal of Personality and Social Psychology, 37, 1859-1870.

Carver, C. S., \& Scheier, M. F. (1982). Control theory: A useful conceptual framework for personality-social, clinical, and health psychology. Psychological Bulletin, 92, 111-135.

Dixon, T. M., \& Baumeister, R. F. (1991). Escaping the self: The moderating effect of selfcomplexity. Personality and Social Psychology Bulletin, 17, 363-368.

Duval, S., \& Wicklund, R. (1972). A theory of objective self-awareness. New York: Academic Press.

Duval, T., Duval, V., \& Mullilis, J. (1992). Effects of self-focus, discrepancy between self and standard, and outcome expectancy favorability on the tendency to match self to standard and withdraw. Journal of Personality and Social Psychology, 62, 340-348.

Dweck, C. S., Hong, Y., \& Chiu, C. (1993). Implicit theories: Individual differences in the likelihood and meaning of dispositional inference. Personality and Social Psychology Bulletin, 19, 644-656.

Forster, J., Grant, H., Idson, L. C., \& Higgins, E. T. (2001). Success/failure feedback, expectancies, and approach/avoidance motivation: How regulatory focus moderates classic relations. Journal of Experimental Social Psychology, 37, 253-260.

Gailliot, M. T., \& Baumeister, R. F. (2007). The physiology of willpower: Linking blood glucose to self-control. Personality and Social Psychology Review, 11, 303-327. 
Higgins, E. T. (1987). Self-discrepancy: A theory relating self and affect. Psychological Review, 94, 319-340.

Hirt, E. R., Deppe, R. K., \& Gordon, L. J. (1991). Self-reported versus behavioral selfhandicapping: Empirical evidence for a theoretical distinction. Journal of Personality and Social Psychology, 61, 981-991.

Isen, A. M. (1987). Positive affect, cognitive processes, and social behavior. In L. Berkowitz (Ed.), Advances in experimental social psychology (Vol. 20, pp. 203-253). New York: Academic Press.

Linville, P. W. (1985). Self-complexity and affective extremity: Don't put all of your eggs in one cognitive basket. Social Cognition, 3, 94-120.

Markus, H., \& Nurius, P. (1986). Possible selves. American Psychologist, 41, 954-969.

McConnell, A. R., Renaud, J. M., Dean, K. K., Green, S. P., Lamoreaux, M. J., Hall, C. E., et al. (2005). Whose self is it anyway? Self-aspect control moderates the relation between selfcomplexity and well-being. Journal of Experimental Social Psychology, 41, 1-18.

McConnell, A. R., \& Strain, L. M. (2007). Content and structure of the self. In C. Sedikides \& S. Spencer (Eds.), The self in social psychology (pp. 51-72). New York: Psychology Press.

Niedenthal, P. M., Setterlund, M. B., \& Wherry, M. B. (1992). Possible self-complexity and affective reactions to goal-relevant evaluation. Journal of Personality and Social Psychology, 63, 5-16.

Pacini, R., \& Epstein, S. (1999). The relation of rational and experiential information processing styles to personality, basic beliefs, and the ratio-bias phenomenon. Journal of Personality and Social Psychology, 76, 972-987.

Phillips, A. G., \& Silvia, P. J. (2005). Self-awareness and the emotional consequences of selfdiscrepancies. Personality and Social Psychology Bulletin, 31, 703-713.

Rafaeli-Mor, E., Gotlib, I. H., \& Revelle, W. (1999). The meaning and measurement of selfcomplexity. Personality and Individual Differences, 27, 341-356.

Renaud, J. M., \& McConnell, A. R. (2002). Organization of the self-concept and the suppression of self-relevant thoughts. Journal of Experimental Social Psychology, 38, 79-86.

Renaud, J. M., \& McConnell, A. R. (2007). Wanting to be better but thinking you can't: Implicit theories of personality moderate the impact of self-discrepancies on self-esteem. Self and Identity, 6, 41-50.

Schleicher, D. J., \& McConnell, A. R. (2005). The complexity of self-complexity: An associated systems theory approach. Social Cognition, 23, 387-416.

Schwarz, N., \& Clore, G. L. (2003). Mood as information: 20 years later. Psychological Inquiry, 14, 296-303.

Scott, W. A. (1969). Structure of natural cognitions. Journal of Personality and Social Psychology, 12, 261-278.

Showers, C. (1992). Compartmentalization of positive and negative self-knowledge: Keeping bad apples out of the bunch. Journal of Personality and Social Psychology, 62, 1036-1049.

Strauman, T. J., \& Higgins, E. T. (1987). Automatic activation of self-discrepancies and emotional syndromes: When cognitive structures influence affect. Journal of Personality and Social Psychology, 53, 1004-1014.

Strube, M. J. (1986). An analysis of the self-handicapping scale. Basic and Applied Social Psychology, 7, 211-224.

Tice, D. M., Bratslavasky, E., \& Baumeister, R.F. (2001). Emotional distress regulation takes precedence over impulse control: If you feel bad, do it! Journal of Personality and Social Psychology, 80, 53-67.

Vohs, K. D., \& Ciarocco, N. J. (2004). Interpersonal functioning requires self-regulation. In R. F. Baumeister \& K. D. Vohs (Eds.), Handbook of self-regulation: Research, theory, and applications (pp. 392-407). New York: Guilford Press.

Watson, D., Clark, L. A., \& Tellegen, A. (1988). Development and validation of brief measures of positive and negative affect: The PANAS scales. Journal of Personality and Social Psychology, 54, 1063-1070. 